\title{
Control effect analysis for arch auxiliary system on long term deflection of long span prestressed concrete continuous beam bridge
}

\author{
J D Zhang ${ }^{1}$, X X Wang ${ }^{1}$, Z Y Lu ${ }^{1}$ \\ ${ }^{1}$ Key Laboratory for Bridge and Tunnel of Shannxi Province, Chang'an University, Xi'an, PR China
}

\begin{abstract}
In order to explore the control effect of arch assisted system on long-term deflection of long-span PC continuous girder bridge, an evaluation framework of long-term deflection control effect is proposed. Firstly, the functional relationship between long-term deflection and structural parameters is fitted based on the measured data, and the deflection prediction model is established; Secondly, based on the calculation method of bending stiffness of inversion theory, the evaluation framework of long-term deflection control effect based on the analogy stiffness method is proposed; Finally, based on the engineering example, under the premise of equal load sharing ratio, the above frame is applied to carry out parameter analysis. The results show that the deflection prediction model based on the measured data has good correlation, and the established evaluation framework can quantitatively evaluate the control effect of arch auxiliary system on long-term deflection.
\end{abstract}

\section{Introduction}

Concrete continuous beam is a bridge type with strong comparability in economic rationality and process maturity. However, its long-term down deflection not only affects the comfort of driving, but also brings serious safety hazards. Ballastless track bridge beam ${ }^{[1]}$, which is widely used in high-speed railway transportation, is difficult to adjust the deformation in time. Therefore, there are more strict requirements for long-term deformation. The research of Gou ${ }^{[2]}$ and so on found that the track deformation is proportional to the deformation of main beam. In order to ensure smooth driving, the vertical deformation of main beam must be controlled; Yang [3] research found that creep deformation can cause obvious impact at the beam end, which is not conducive to highspeed driving. In order to control the down deflection of long-span PC continuous beam bridge, the traditional methods such as prestress preparation beam ${ }^{[4]}$ are the early attempts of engineers. However, it is difficult to apply the prestressed beam because of its low efficiency. The long-span concrete continuous beam arch composite system provides a solution to the above problems ${ }^{[5]}$. Dai et al. ${ }^{[6]}$ compares the interaction characteristics between continuous beam and beam rail, it is shown that the arch auxiliary long-span PC continuous beam bridge has the advantages of large rigidity, smooth driving, large bearing capacity and simple maintenance. However, there is little research on how to evaluate the effect of long-term over down control measures.
This paper takes a long-span arch auxiliary PC continuous beam bridge as an example to analyze the control effect and evaluate the effect of long-term over deflection control measures. Firstly, based on the measured data, the response surface method is used to fit the function relationship between long-term deflection and structural parameters, and a long-term down deflection prediction model is established; Secondly, based on the inversion theory, the calculation method of bending stiffness of beam body is proposed, and the evaluation framework of long-term deflection control effect based on the method of analogy stiffness is proposed; Finally, based on the engineering example, the parameters analysis is carried out by using the above frame under the premise of equal load sharing ratio, and the long-term down deflection control effect of arch auxiliary system is quantitatively evaluated, and the influence law of long-term down deflection of long-term continuous beam system with large span arch auxiliary type is revealed, which provides reference for designers in the initial structure selection.

\section{Long term deflection prediction model of long span PC Continuous Girder Bridge Based on measured data}

At present, the most common method in engineering field is to fit some empirical formulas to predict the long-term deflection of structures according to the engineering investigation and test. But the empirical formula predicts the long-term deflection of the structure is based on the ideal structure. The actual bridge structure is coupled with 
crack and prestress loss during operation and maintenance [7], which makes the actual structure deviate from the ideal state. The traditional empirical formula considers single factors, can not consider coupling effects of many nonlinear factors at the same time, and the prediction error is large. In order to reflect the real service condition of the structure as much as possible and reduce the calculation error in the process of parameter determination, this paper forecasts and analyzes the long-term deflection of PC continuous beam from the aspect of span and girder height by measuring the long-term deflection data of the bridge formed by different span beams from 5 to 10 years later.

The response function of the actual structure is difficult to express explicitly, which brings difficulties to the structural analysis. Response surface method ${ }^{[8]}$ can approximate the real function of structure by fitting quadratic surface, which has high efficiency and can meet the requirements of engineering precision. The common response surface method is an iterative sequence response surface method to obtain the checking point, as shown in formula (1):

$$
y=\beta_{0}+\sum_{i=1}^{K} \beta_{i} x_{i}+\sum_{i} \sum_{j} \beta_{i j} x_{i} x_{j}+\sum_{i=1}^{K} \beta_{i i} x_{i}^{2}
$$

Where: $x_{i} \in\left[x_{i}^{I}, x_{i}^{U}\right], x_{i}^{I}, x_{i}^{U}$ is the value range of design parameters; $\beta_{0}, \beta_{i}, \beta_{i j}, \beta_{i i}$ is the regression coefficient.

Table1. Long term deflection measured data sheet

\begin{tabular}{cccc}
\hline Bridge name & $\begin{array}{c}\text { Main } \\
\text { spans (m) }\end{array}$ & $\begin{array}{c}\text { Height of main } \\
\text { beam (m) }\end{array}$ & $\begin{array}{c}\text { Long term } \\
\text { deflection } \\
(\mathrm{cm})\end{array}$ \\
\hline $\begin{array}{c}\text { Liuguang River } \\
\text { Bridge }\end{array}$ & 240 & 4.1 & 56 \\
$\begin{array}{c}\text { Parrotts Ferry } \\
\text { Bridge }\end{array}$ & 195 & 3.2 & 64 \\
$\begin{array}{c}\text { Grand mere } \\
\text { Bridge }\end{array}$ & 181.4 & 2.9 & 30 \\
$\begin{array}{c}\text { Dongpu Bridge } \\
\text { Auxiliary bridge } \\
\text { of Yaji Bridge } \\
\text { South lutrive } \\
\text { Bridge }\end{array}$ & 160 & 3.2 & 23 \\
Sanmenxia & 160 & 2.5 & 26 \\
Yellow River \\
$\begin{array}{c}\text { Bridge } \\
\text { Nanhai Jinsha } \\
\text { Bridge }\end{array}$ & 140 & 2.2 & 16 \\
Nhvv (unit 3-ib) \\
bridge
\end{tabular}

Based on the measured data in table 1), through design expert 10.0, the relationship between girder span, girder height and long-term deflection is fitted, as shown in formula (2). $f=-280.6-4.1 \times A+325.18 \times B+2.36 \times A B-0.0144 \times A^{2}-91.77 \times B^{2}$

Where: $f$ is the long-term deflection value; $A$ is the span length, $B$ is the height of the main beam.

\section{Evaluation framework of long term deflection control effect based on analogy stiffness method}

Due to the coupling effect of various factors, the crosssection of service bridge cracks and its stiffness decreases, and its long-term deflection is often much larger than the theoretical calculation value. Therefore, in order to simulate the real state of the structure, it is necessary to reduce the structural stiffness.

\subsection{Calculation formula of equivalent elastic modulus}

The bending stiffness of the member can be expressed as the product of elastic modulus $E_{c}$ and section moment of inertia $I_{0}$. For reinforced concrete flexural members, the effective moment of inertia method [9] is used to calculate the bending stiffness of the section. Before concrete cracking, the area of steel bar in tension and compression zone is $A_{s}$ and $A_{s}^{\prime}$ respectively, which is converted into the cross-sectional areas are $n A_{s}$ and $n A_{s}^{\prime}$ respectively, where $n=E_{s} / E_{c}$. Add an additional area $(n-1) A_{s}$ and $(n-1) A_{s}^{\prime}$ at the position of the reinforcement position, as shown in figure 1.

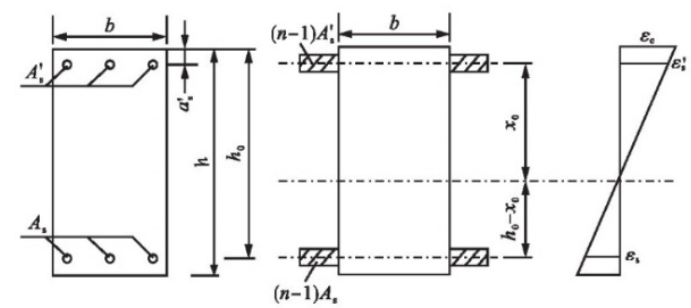

Fig 1. Conversion section of beam

The converted moment of inertia of the cross section is:

$$
I_{0}=\frac{b}{3}\left[x_{0}^{3}+\left(h-x_{0}\right)^{3}\right]+(n-1) A_{s}\left(h_{0}-x_{0}\right)^{2}+(n-1) A_{s}^{\prime}\left(x_{0}-a_{x}^{\prime}\right)^{2}
$$

\subsection{Analogy stiffness method based on finite element model}

In this paper, the "indirect method ${ }^{[10] "}$ is used to calculate the structural stiffness reduction factor, and the influence of materials on the long-term deflection of the structure is ignored, the structural stiffness reduction only reduces the moment of inertia of the section. 


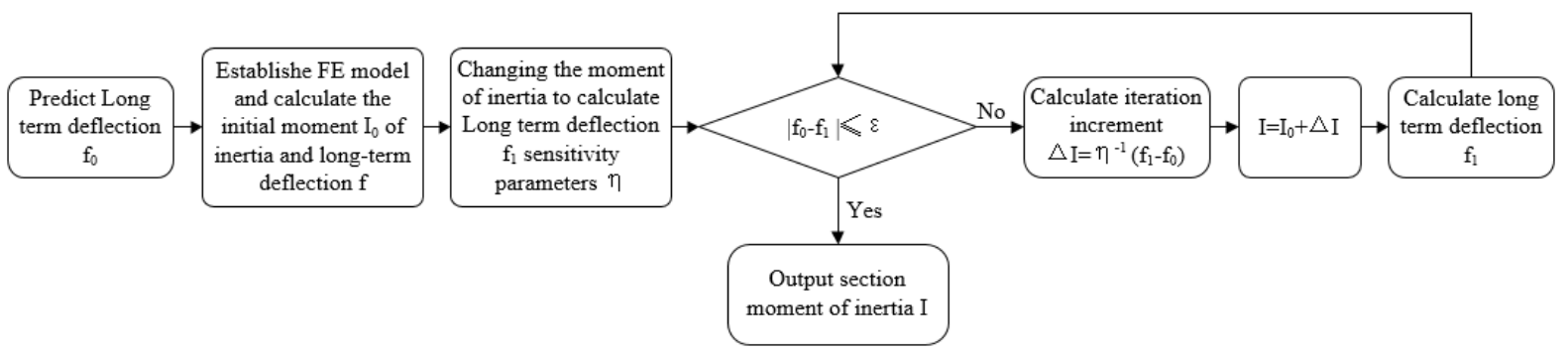

Fig 2. Flow chart of analogy stiffness method

The basic theory of the indirect method is that the moment of inertia of the beam is regarded as a variable. By changing the value of the moment of inertia of the structure in the finite element model, the error between the calculated result and the predicted value can meet the requirements. The program flow chart is shown in figure 2. The initial value of inertia moment of all elements is taken as the design value and the calculation is started. In the second calculation, an increment of inertia moment of the section is given, and then a finite element analysis is made. The long-term deflection difference obtained from the two calculations divided by the increment of inertia moment of the section is the sensitivity parameter $\eta$ of torsion to inertia moment.

In order to explore the control effect of arch auxiliary system on long-term deflection of long-span PC, the finite element model of continuous beam is established firstly. Through the above flow chart, the reduced section moment of inertia can be obtained, so that the long-term deflection of the calculation model can reach the predicted value. On the basis of constant parameters of continuous beam, the arch auxiliary structure is applied, and the improvement effect of long-term deflection by adding arch structure is compared and analyzed, as shown in figure 3 .

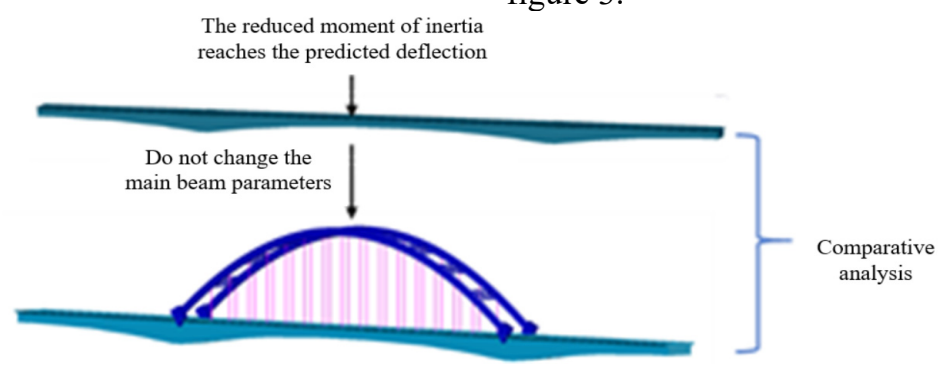

Fig 3. Analogy stiffness method for long term deflection

\section{Case study}

\subsection{Engineering background}

This paper takes the prestressed concrete continuous beam bridge with span of $76 m+160 m+76 m$ as the research object. As shown in figure 4, the main beam adopts C55 concrete, adopts box section with single box and double chamber variable height, the mid span beam is $3.5 \mathrm{~m}$ high, the beam height at the fulcrum is $8.5 \mathrm{~m}$, the beam bottom is changed according to the circular curve, the section of the main beam of arch auxiliary structure is the same as that of continuous beam, and the arch rib is dumbbell shaped section. The calculated span is $160 \mathrm{~m}$ and the sagittal height is $32 \mathrm{~m}$, the ratio of midsagittal span is $1 / 5$, the section height is $3 \mathrm{M}$, the diameter of arch rib chord is $1 \mathrm{~m}$, and it is connected by $16 \mathrm{~mm}$ thick steel infilled plate, and the arch rib chord and infilled with micro expansion concrete. Q345 steel is used for steel pipe, and C55 shrinkage compensating concrete is used in steel pipe; There are 15 groups of double suspenders in the whole bridge. The main bridge adopts the construction method of "beam before arch", and the track is laid 6 months after the completion of the bridge.

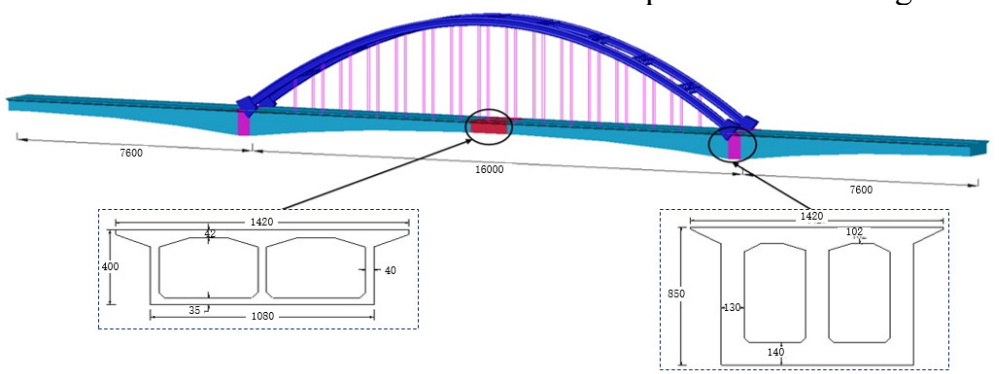

Fig 4. General layout ( $\mathrm{cm})$

In the finite element model, the main beam and arch rib are simulated by spatial beam element, the suspender is simulated by truss element, the concrete-filled steel tubular arch is simulated by construction joint section, the suspender and the main beam and the arch foot and the main beam are rigidly connected by master-slave node, the cross brace and arch rib are rigidly connected by elastic connection, the pier top is fixed support, and other supports are movable support, There are 610 nodes and 859 elements in the arch auxiliary structure, and 120 
nodes and 118 elements in the continuous beam finite element model.

\subsection{Control effect analysis and evaluation}

Taking the completed bridge state as the initial state, the long-term deflection values of arch assisted composite bridge and continuous beam bridge are compared and analyzed ten years after the completion of the bridge. Based on the above long-term deflection prediction model, the long-term deflection value of the bridge is $26.02 \mathrm{~cm}$. The long-term deflection value of the structure reaches the predicted value through the analogy stiffness method, and the arch auxiliary structure is applied on the basis of the continuous beam to obtain the long-term deflection of the continuous beam and arch auxiliary structure, as shown in Figure 5.

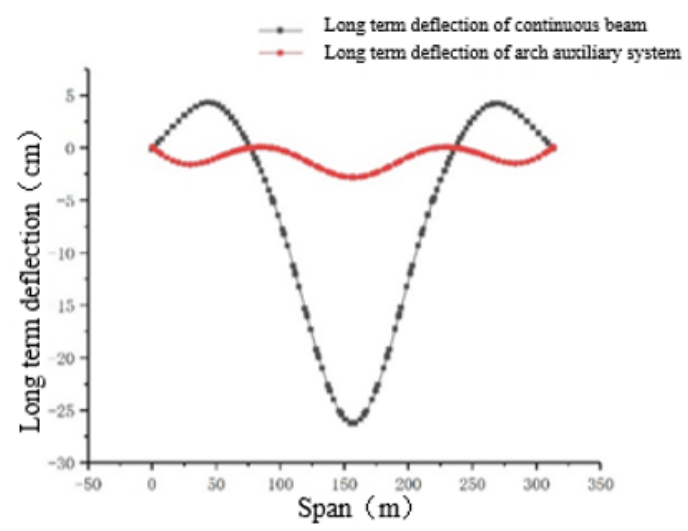

Fig 5. Comparison chart of long term deflection

It can be seen from Figure 7 that the arch auxiliary system improves the structural stiffness. The mid span deflection is reduced from $26.02 \mathrm{~cm}$ to $2.83 \mathrm{~cm}$, the upper deflection of side span and middle span is reduced from $4.3 \mathrm{~cm}$ to $1.24 \mathrm{~cm}$.

In order to further explore the improvement effect of arch auxiliary structure on long-term deflection of different span and different beam height, based on the load sharing ratio formula proposed in literature ${ }^{[11]}$, the same load sharing ratio is maintained. The improvement effect of arch auxiliary structure on long-term deflection of continuous beam with different span and different beam height is compared and analyzed.

$$
t=\frac{k_{s a}\left[4 \eta\left(1+k_{a}\right)\right]+k_{b a}\left(1+6 k_{a}+4 \eta+4 k_{a} \eta\right)}{k_{s a}\left[4 \eta\left(1+k_{a}\right)+k_{b a}\left(1+6 k_{a}+4 \eta+8 k_{a} \eta\right)+2 k_{a} k_{b a}^{2}(3+2 \eta)\right]+k_{f} k_{b a}\left[1536 \eta\left(1+k_{a}\right)+128 k_{b a}\left(3+18 k_{a}+2 \eta+12 k_{a} \eta\right)\right]}
$$

Where: $t$ is the ratio of load sharing of beam arch; $k_{b a}=\frac{E_{b} I_{b}}{E_{a} I_{a}}$ is the ratio of flexural stiffness of beam arch section; $k_{f}=\frac{f}{L}$ is the ratio of arch rib to vector Quart; $k_{s a}=\frac{e a_{s} L^{3}}{E_{a} I_{a}}$ is the ratio of stiffness of arch rib of suspender; $e a_{s}=\frac{E_{s} A_{s}}{a}$ is the axial stiffness of suspender evenly distributed; $E_{s} A_{s}$ is the axial tension and compression stiffness of suspender; $a$ is spacing between suspenders.

\subsection{Influence of span length and beam height on long term deflection}

In order to explore the improvement effect of arch auxiliary structure on the long-term deflection of continuous beams with different spans, four prestressed continuous beam bridges with different spans in table 2) are used to fit the long-term deflection with the above prediction model, and the load sharing ratio after applying arch auxiliary structure is calculated.
Table2. Deflection and load sharing ratio with different

\begin{tabular}{ccc}
\multicolumn{3}{c}{ span } \\
\hline span & deflection $(\mathrm{cm})$ & load sharing ratio \\
\hline $140 \mathrm{~m}$ & -15.6 & 0.963 \\
$160 \mathrm{~m}$ & -26.02 & 0.966 \\
$180 \mathrm{~m}$ & -30.8 & 0.968 \\
$200 \mathrm{~m}$ & -37.9 & 0.970 \\
\hline
\end{tabular}

It can be seen from table 2 that the load sharing ratio of the four bridges is close to 1 after the arch auxiliary structure is applied. On this basis, the long-term deflection value of the structure is calculated. It can be seen from the figure 6 that the long-term deflection of the continuous beam increases with the increase of the span, and the longterm deflection increases about $19.5 \%$ for every $20 \mathrm{~m}$ increase of the span. After the arch auxiliary structure is applied, the long-term deflection of the structure decreases significantly, with an average decrease of $88.2 \%$, With the increase of span, the long-term deflection of the structure increases slowly, and the long-term deflection increases about $14.3 \%$ with the increase of 20 $\mathrm{m}$ span. It can be seen that the arch assisted system can significantly reduce the long-term deflection of the structure. 


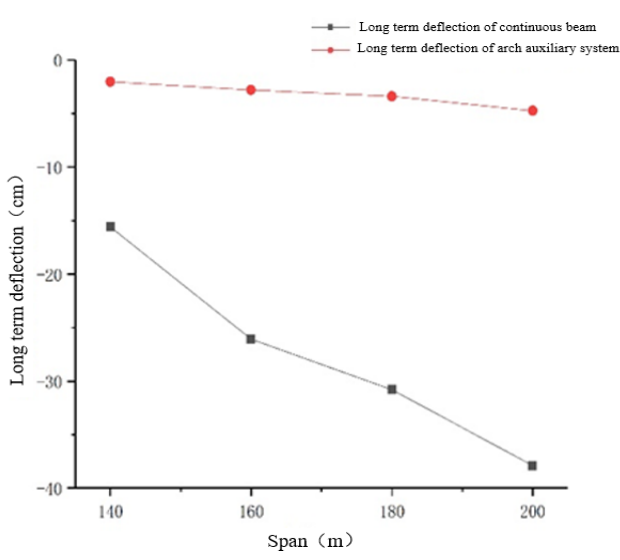

Fig 6. Long term deflection diagram of main span with different span

The most direct way to improve the structural stiffness of PC continuous beam is to increase the main beam height, but the structural weight will also increase. In order to explore the influence of the main beam height on the long-term deflection of the arch auxiliary system, take the background bridge with a main span of $160 \mathrm{~m}$ as an example, adjust the main beam height respectively, use the above model to predict the long-term deflection, and calculate the load sharing after applying the arch auxiliary structure, as shown in table 3).

Table3. Deflection and load sharing ratio with different beam height

\begin{tabular}{ccc}
\hline beam height & deflection $(\mathrm{cm})$ & load sharing ratio \\
\hline $3.5 \mathrm{~m}$ & -27.36 & 0.975 \\
$4 \mathrm{~m}$ & -26.07 & 0.976 \\
$4.5 \mathrm{~m}$ & -25.05 & 0.974 \\
$5 \mathrm{~m}$ & -23.65 & 0.973 \\
\hline
\end{tabular}

It can be seen from table 4 that the load sharing ratio of the four bridges is close to 1 after applying the auxiliary arch system. On this basis, the long-term deflection value of the auxiliary arch composite bridge with different beam heights is calculated as shown in figure 7 . It can be seen from the figure that the increase of the main beam height has a significant effect on the long-term deflection of the continuous beam. When the beam height increases from $3.5 \mathrm{~m}$ to $5 \mathrm{~m}$, the long-term deflection of the continuous beam decreases by $12.6 \%$. When the beam height is $4 \mathrm{~m}$, the improvement effect of applying the arch assisted system on the long-term deflection is the most obvious, but the improvement effect of increasing the main beam height on the long-term deflection of the arch assisted composite bridge is not obvious.

\section{Conclusion}

(1) The long-term deflection prediction function of continuous beam bridge based on measured data has good correlation and practicability.

(2) The long-term deflection of continuous beam bridge is more sensitive to the change of main beam height and span. For continuous beams with different spans, selecting appropriate main beam height can effectively reduce the long-term deflection.

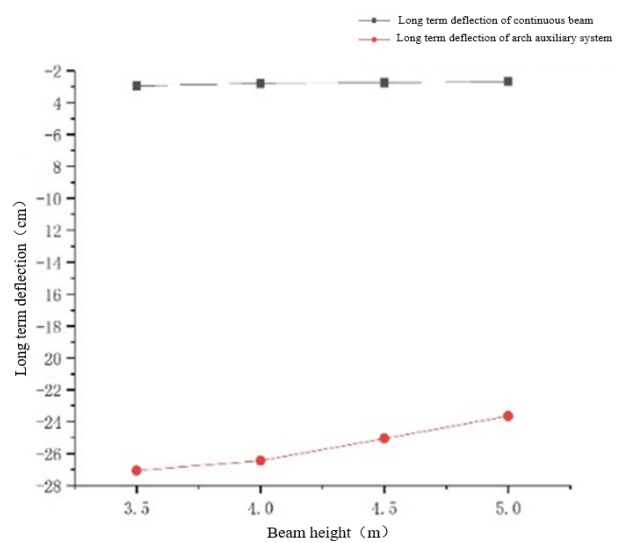

Fig 7. Long term deflection diagram of main span with different beam height

(3) For the background bridge, the proposed evaluation framework can quantitatively evaluate the improvement effect of arch assisted system on long-term deflection.

(4) For continuous beams with different spans and girder heights, the long-term deflection of continuous beams can be significantly reduced by applying arch auxiliary structure. The long-term deflection of continuous beams with different spans and girder heights can be reduced by $88.2 \%$ and $89.1 \%$ respectively.

(5) For arch auxiliary composite bridge with similar load sharing ratio, the change of main beam height and span has little influence on the long-term deflection of the structure.

\section{References}

1. Xin Tao et al. 2016 Dynamic influence and structural optimization of damping cushion for CRTs III slab ballastless track of high speed railway China Railway Science. 37 1-7

2. Gou Hongye et al. 2018 Study on the influence of bridge vertical deformation on track smoothness Journal of railway engineering. 35 44-49

3. YANG H et al. 2014 Dynamic Analysis of TrainRail-Bridge Interaction Considering Concrete Creep of a Multi-Span Simply Supported Bridge Advances in Structural Engineering. 175

4. HANBING Z et al. 2015 External Prestressing Bridge Reinforcement Technology Review Conf. MATEC.

5. Yang shankui et al. 2013 Design of continuous beam arch composite structure of Railway Passenger Dedicated Line Journal of railway engineering. 30 48-52

6. Dai Gonglian et al. 2017 Comparison of beam rail interaction between long span continuous beam bridge and beam arch composite bridge Journal of Central South University (NATURAL SCIENCE EDITION). 48 233-238

7. Li Xiaoxiang et al. 2006 Study on long-term deflection of main girder of long-span prestressed concrete girder bridge Conf. Proceedings of the 
National Conference on prestressed structural theory and engineering application

8. Tian Hao et al. 2011 Time dependent reliability analysis of concrete bridge based on response surface method Journal of Tongji University (NATURAL SCIENCE EDITION). 39 166-171

9. Guo Zhenhai et al. 2003 Principle And Analysis of Reinforced Concrete.

10. Lin Xinyuan. 2010 Research on mechanical behavior of prestressed concrete continuous box girder with variable cross section after cracking (Chang'an University)

11. [11] Chen Hailang. 2011 Research on Cooperative mechanical characteristics of composite bridge members (Zhejiang University) 